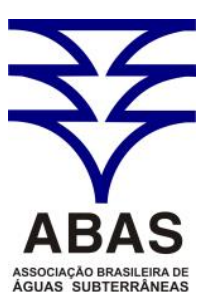

ASSOCIACAO BRASLLERADE
AGGUAS SUBTERRANEAS www.abas.org

\title{
INFLUÊNCIA DA TAXA DE DOSE POTENCIAL VARIÁVEL EM ÁREAS CONTAMINADAS NO CÁLCULO DO RISCO À SAÚDE HUMANA
}

\author{
INFLUENCE OF VARIABLE POTENTIAL DOSE RATE AT \\ CONTAMINATED AREAS ON THE CALCULATIONS OF HUMAN HEALTH \\ RISK
}

\author{
Karina Lopes Joussef ${ }^{1}$, Cristina Cardoso Nunes², Elizabeth May Pontedeiro ${ }^{3}$, Mário do \\ Rosário ${ }^{4}$, Henry Xavier Corseuil ${ }^{5}$
}

Artigo recebido em: 10/10/2013 e aceito para publicação em: 28/11/2013.

\begin{abstract}
In the management of contaminated sites, traditional methodologies for human health risk assessment (e.g., RBCA) assume that the receptors are exposed to a maximum and constant concentration, obtained with a single environmental sampling throughout the entire time of exposure ( 25 - 30 years). This study aims to evaluate the influence of temporal variability on the quantification of human health risk using variable contaminant concentrations. The data pertained to a field experiment with controlled release of Brazilian gasoline from its initial release in groundwater until complete attenuation. Experimental data from the field site shows that the maximum exposure concentrations of benzene and toluene occurred during a short period of the 25 years during which exposure was considered. The conventional approach of risk calculation was found to produce results varying by two orders of magnitude depending upon the sampling period. Having a complementary procedure for quantifying risk focusing on variability in the potential dose rate in models that simulate transport and transformation processes may provide risk values that are more consistent with site-specific conditions. This will reduce uncertainties in the quantification of risk with regard to representative concentrations in the area, thus improving decision-making technologies for remediating impacted areas.
\end{abstract}

Keywords: Management of contaminated sites. Human health risk assessment. Natural attenuation. Variable potential dose rate.

Resumo: No gerenciamento de áreas contaminadas, as metodologias tradicionais de avaliação de risco à saúde humana (i.e., RBCA) assumem que os receptores estão expostos a uma concentração máxima e constante, obtida em um único monitoramento ambiental, ao longo de toda a duração de exposição (25-30 anos). Por isso, este trabalho tem como objetivo avaliar a influência da temporalidade na quantificação do risco à saúde humana, considerando a variação das concentrações dos contaminantes. Os dados utilizados são de um experimento de campo com liberação controlada de gasolina brasileira, desde a sua liberação na água subterrânea até a sua completa atenuação. Os dados experimentais da área em estudo mostram que as máximas concentrações de exposição de benzeno e tolueno ocorreram durante um curto período, se considerado uma duração de exposição de 25 anos. A abordagem convencional de cálculo de risco apresentou valores de risco variando em duas ordens de grandeza, dependendo do período amostrado. Utilizando um procedimento complementar de quantificação de risco com foco na variação da taxa de dose potencial em modelos que simulem os processos de transporte e transformação do contaminante, pode-se obter valores de risco de acordo com as condições específicas do local. Isto pode reduzir as incertezas na quantificação do risco no que se refere à escolha da conc entração representativa da área, e auxiliar na definição de tecnologias mais adequadas para recuperação de áreas impactadas.

Palavras-chave: Gerenciamento de áreas contaminadas. Avaliação de risco à saúde humana. Atenuação natural. Taxa de dose potencial variável.

\section{INTRODUÇÃO}

No processo de gerenciamento ambiental de áreas contaminadas ou de áreas com atividades potencialmente poluidoras, a avaliação de risco à saúde humana representa uma importante etapa na

\footnotetext{
${ }^{1}$ Universidade Federal de Santa Catarina (kajoussef@gmail.com).

2 Universidade Federal de Santa Catarina (crisccnunes@gmail.com).

${ }^{3}$ Universidade Federal do Rio de Janeiro (bettinadulley@hotmail.com).

${ }^{4}$ Universidade Federal do Rio de Janeiro (marioros@ petrobras.com.br).

${ }^{5}$ Universidade Federal de Santa Catarina (henry.corseuil@ufsc.br).
} 
caracterização dos potenciais efeitos de substâncias tóxicas sobre a saúde de indivíduos ou populações (NRC, 1983). Prevista na Resolução $n^{\circ}$ 420/2009 do Conselho Nacional de Meio Ambiente - CONAMA (CONAMA, 2009), a tomada de decisão baseada no risco permite avaliar a necessidade e priorizar as ações corretivas adequadas em função do risco real ou potencial, estabelecendo valores orientadores e metas de remediação específicas para o sítio impactado (USEPA, 1989a; CETESB, 2006).

As referências técnico-científicas mais conhecidas para a avaliação de risco à saúde humana em locais contaminados com produtos químicos e de derivados de petróleo estão reunidas na metodologia Risk-Based Corrective Action - RBCA da American Society for Testing and Materials ASTM, através dos documentos ASTM E173995-Reapproved 2010 (ASTM, 2010) e ASTM E2081-00 (ASTM, 2000). A metodologia RBCA foi elaborada conforme orientações da United States Environmental Protection Agency - USEPA (USEPA, 1989a), sendo composta por uma sequência lógica de procedimentos e decisões, organizada em níveis. O primeiro nível aponta para a utilização de padrões genéricos estabelecidos pela legislação, seguido pelos níveis mais complexos, com coleta de dados e análise, de acordo com a especificidade do local (ASTM, 2000, 2010). No Brasil, a Companhia Ambiental do Estado de São Paulo - CETESB, instituiu em 2006 o procedimento Ação Corretiva Baseada no Risco - ACBR (CETESB, 2006), tomando como base a metodologia RBCA.

A quantificação do risco depende da caracterização da exposição humana a compostos químicos presentes no meio físico, determinada através das concentrações de exposição das substâncias que oferecem risco à saúde humana nas áreas potencialmente contaminadas. Para a definição da concentração provável com a qual o receptor pode entrar em contato ao longo da duração de exposição, utiliza-se tanto a concentração média dos valores de concentrações coletados, como o limite superior de confiança ( $95 \%$ ) da média (95 percent Upper Confidence Limit - UCL95), ou a concentração máxima para o ponto de exposição (USEPA, 1989a, 1992a, 2002; CETESB, 2001). Dadas as limitações apresentadas para o uso de uma concentração média representativa da área impactada, o uso da concentração máxima obtida em um único monitoramento ambiental é comumente utilizado para a quantificação do risco. No entanto, a concentração máxima medida durante a amostragem ambiental nem sempre é conservadora, pois na etapa de investigação detalhada o mo- nitoramento periódico do meio não é realizado, e $\operatorname{logo}$, não se tem outras medições que confirmem se a concentração máxima escolhida corresponde à máxima concentração que poderia ocorrer na área.

A modelagem matemática é um instrumento de apoio nas avaliações de risco, no qual modelos de transporte e transformação são requeridos para estimar futuras concentrações em pontos de exposição não contaminados ou em pontos onde não existem dados de monitoramento (USEPA, 1989a; CETESB, 2001). Entretanto, mesmo quando modelos matemáticos (Bioscreen (NEWELL et al., 1996), RISC (SPENCE e WALDEN, 2001), RBCA Tool Kit (CONNOR et al., 2007), SCBR (CORSEUIL et al., 2006; COLONESE, 2010; SCHNEIDER et al., 2012)), que simulam os fenômenos naturais, são utilizados para obter concentrações ao longo do tempo em pontos de exposição de interesse, a escolha de um único valor é mantida para o cálculo do risco ao se utilizar a metodologia tradicional (USEPA, 1989a, 1992a). Isso indica que, sejam por dados monitorados ou simulados, essas metodologias supõem que os receptores estarão expostos a uma concentração constante ao longo de toda a duração de exposição ( 25 ou 30 anos, dependendo do tipo de receptor e uso do solo).

Nas metodologias de avaliação de risco de materiais radioativos (IAEA, 2004a; IAEA, 2004b) é usual a utilização de modelos de transporte para prognosticar a variação das concentrações dos radionucleotídeos ao longo do tempo no meio ambiente. A partir das concentrações simuladas, pode-se calcular a dose anual e/ou risco anual radiológico como uma função do tempo, e comparar os resultados obtidos temporalmente com os limites estabelecidos pelas legislações (IAEA, 1999; CNEN, 2005; PONTEDEIRO et al., 2007, 2010). Sob a mesma ótica utilizada na análise de risco de rejeitos radioativos, alguns estudos têm aprofundado a influência da temporalidade das concentrações na toxicidade e no risco de substâncias químicas ambientais (BIÉN et al., 2004; CHEN, 2009; BACIOCCHI et al., 2010; ANDRICEVIC et al., 2012). O objetivo deste trabalho é apresentar um estudo de caso de um experimento de campo com liberação controlada de gasolina brasileira (contendo $24 \%$ de etanol) e monitorado durante 14 anos (CORSEUIL et al., 2011) para avaliar a influência da temporalidade na quantificação do risco à saúde humana, considerando a variação das concentrações dos contaminantes desde a sua liberação na água subterrânea até a sua completa atenuação. $\mathrm{O}$ estudo de caso utilizou como rota de exposição a ingestão 
de água subterrânea. No entanto, os conceitos aqui discutidos são aplicáveis para outras rotas de exposições a partir de água subterrânea e solos contaminados.

\section{MATERIAIS E MÉTODOS}

\section{Estudo de Caso}

O experimento de liberação controlada de gasolina com etanol está localizado no sul da cidade de Florianópolis (SC), na Fazenda Experimental da Ressacada, e teve início em 1998 com a liberação de $100 \mathrm{~L}$ de gasolina com $24 \%$ de etanol em uma cava no solo de 1,5 m de largura x $1,0 \mathrm{~m}$ de comprimento $\mathrm{x} 1,5 \mathrm{~m}$ de profundidade. $\mathrm{O}$ combustível foi adicionado, em um único pulso, após o rebaixamento de $0,10 \mathrm{~m}$ do nível do lençol freático. A fração média de carbono orgânico da área é de $0,06 \%$, e o solo local é caracterizado como areia argilosa nas camadas superiores e areia fina na subsuperfície com menos de 5\% de silte e argila. O clima regional é mesotérmico úmido, com precipitação anual de $1600 \mathrm{~mm}$. A porosidade efetiva é de $20 \%$ e a direção do fluxo da água subterrânea varia localmente em até $40^{\circ}$. $\mathrm{Na}$ zona saturada, a condutividade média é $1,1 \mathrm{x}$ $10^{-4} \mathrm{~cm} / \mathrm{s}$ e o gradiente hidráulico médio de 0,018 $\mathrm{m} / \mathrm{m}$. A área é composta por 51 poços multiníveis, com 5 níveis cada $(1,0 ; 2,0 ; 2,5 ; 3,5$ e $4,5 \mathrm{~m}$ de profundidade em relação à cota do terreno) instalados na direção preferencial do fluxo subterrâneo (CORSEUIL et al., 2011).
Para o modelo conceitual de exposição da área da Atenuação Natural Monitorada - ANM foram considerados trabalhadores em uso de solo comercial/industrial, expostos à ingestão de água subterrânea contaminada (Tabela 1), e que os mesmos se encontravam na região da fonte. Dentre os hidrocarbonetos monoaromáticos BTEX presentes na gasolina, os compostos benzeno e tolueno foram selecionados para as avaliações de risco. A escolha destas substâncias como compostos químicos de interesse é devida a sua conhecida alta solubilidade relativa em água (AFCEE, 1998) e toxicidade à saúde humana (USEPA 1998, 2005), além de representarem naturezas tóxicas distintas (carcinogênico e não carcinogênico, respectivamente). Os dados temporais de benzeno e tolueno utilizados para quantificação do risco pertencem ao poço de monitoramento PM4, distante 2,6 m à jusante da fonte (sentido sudoeste), e que apresentou as maiores concentrações destas substâncias (nas profundidades de 2,0 e 2,5 m), configurando, assim, um cenário conservador de exposição (pior caso). No último monitoramento realizado no poço PM4 (após 14,3 anos da liberação do combustível), concentrações dissolvidas de benzeno e tolueno estavam abaixo do limite de detecção $(1 \mu \mathrm{g} / \mathrm{L})$ (USEPA, 1993), caracterizando a completa atenuação desses compostos. A distribuição dos poços de monitoramento, a direção preferencial do fluxo e a localização da fonte e do poço PM4 são apresentadas na Figura 1.

Tabela 1 - Parâmetros de exposição para trabalhadores em uso de solo comercial/industrial para a rota de ingestão de água subterrânea

Table 1 - Exposure parameters for workers in soil use commercial/industrial to the route of intake of ground water

\begin{tabular}{cc}
\hline Parâmetros & Valores \\
\hline Taxa de ingestão $^{(1)}(I R)$ & $1 \mathrm{~L} /$ dia \\
Frequência de exposição $^{(1)}(E F)$ & 250 dias/ano \\
Duração de exposição $^{(1)}(E D)$ & 25 anos \\
Peso corpóreo $^{(1)}(B W)$ & $70 \mathrm{~kg}$ \\
Tempo médio para efeitos carcinogênicos ${ }^{(1)}\left(A T_{c}\right)$ & 70 anos \\
Tempo médio para efeitos não carcinogênicos ${ }^{(1)}\left(A T_{n c}\right)$ & 25 anos \\
Fator de carcinogenicidade - benzeno $^{(2)}(S F)$ & $0,055 \mathrm{~kg} . \operatorname{dia} / \mathrm{mg}$ \\
Dose de referência crônica - tolueno $^{(2)}(R f D)$ & $0,08 \mathrm{mg} / \mathrm{kg} \cdot \operatorname{dia}$ \\
\hline
\end{tabular}

Fonte: ${ }^{(1)}$ ASTM, 2010; ${ }^{(2)}$ IRIS, 2009. 

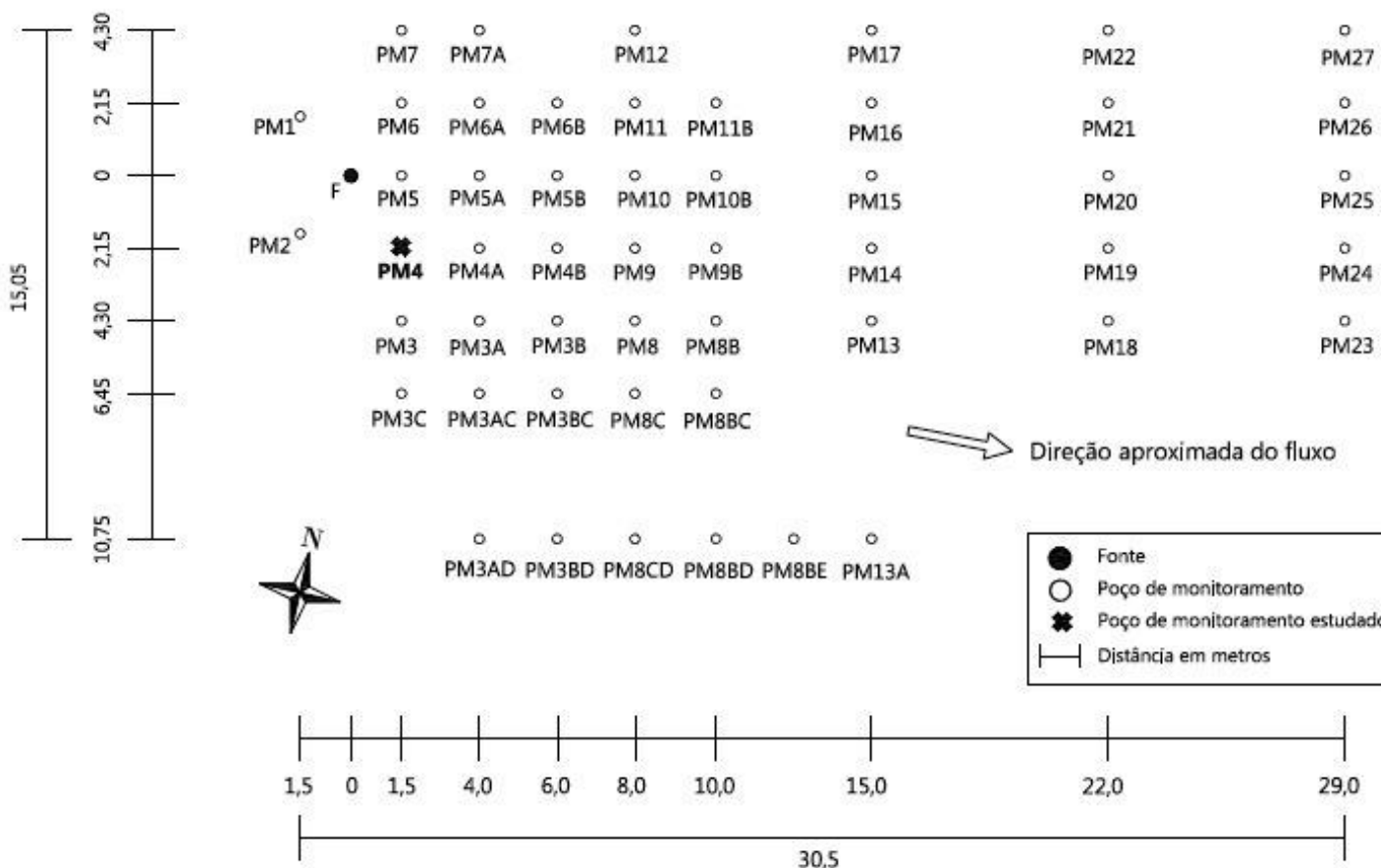

Figura 1 - Representação da área experimental da Atenuação Natural Monitorada (ANM) com sua fonte, poços de monitoramento e direção preferencial do fluxo da água subterrânea (aproximada)

Figure 1 - Representation of the experimental area of Monitored Natural Attenuation (ANM) with its source, monitoring wells and preferred direction of groundwater flow (approximate)

\section{Avaliação de risco à saúde humana}

A metodologia RBCA segue as recomendações da USEPA (USEPA, 1988; USEPA 1989a; USEPA, 1989b; USEPA, 1989c), onde a quantificação do risco assume uma relação linear entre a dose de contaminantes e o risco à saúde do indivíduo, com base no critério da Exposição Máxima Razoável - EMR (USEPA, 1989a; CETESB, 2001; ASTM, 2000, 2010). As doses são frequentemente apresentadas como taxas de dose potencial (mg/ano), ou a quantidade do composto químico ingerido, inalado ou absorvido pela pele (dose potencial) por unidade de tempo (USEPA, 1992b; WHO, 1999). Nas avaliações de risco à saúde humana, as taxas de dose potencial são geralmente expressas como uma dose média diária por unidade de peso corpóreo ( $\mathrm{mg} / \mathrm{kg}$.dia), denominada dose de ingresso (USEPA, 1989a). A equação 1 apresenta a expressão utilizada para o cálculo da dose de ingresso a partir da ingestão de água subterrânea.

$$
I=\frac{C \cdot I R \cdot E F \cdot E D}{B W \cdot A T \cdot 365 \cdot\left(\frac{\text { dias }}{\text { ano }}\right)} \quad \text { (equação 1) }
$$

onde $I$ é a dose de ingresso ( $\mathrm{mg} / \mathrm{kg}$.dia); $C$ é a concentração do contaminante na água subterrânea $(\mathrm{mg} / \mathrm{L}) ; I R$ é a taxa de ingestão de água subterrânea (L/dia); $E F$ é a frequência de exposição (dias/ano); $E D$ é a duração de exposição (anos);
$B W$ é o peso corpóreo $(\mathrm{kg}) ; A T$ é o tempo médio (anos).

Para efeitos carcinogênicos, o risco (R) é estimado a partir do fator de carcinogenicidade (SF) como a probabilidade de um indivíduo desenvolver câncer durante o seu tempo de vida (equação 2). $\mathrm{O}$ risco de substâncias não carcinogênicas é avaliado pela comparação entre a dose de ingresso (I) e a dose de referência (RfD), para uma duração de exposição similar, representada pelo quociente de perigo (HQ) (equação 3). Ainda que a RfD, estimada em curvas de dose-resposta de uma determinada substância química possa estar disponível para vários tempos de exposição considerados pela USEPA (agudo - inferior a 2 semanas; subcrônico - entre 2 semanas e 7 anos; crônico - entre 7 anos e o tempo de vida), neste trabalho optou-se por utilizar o parâmetro toxicológico crônico por fornecer valores de risco não carcinogênicos mais conservadores.

$$
\begin{aligned}
& R=I . S F \\
& H Q=\frac{I}{R f D}
\end{aligned}
$$

(equação 2)

(equação 3)

onde $R$ é o risco carcinogênico (adimensional); $S F$ é o fator de carcinogenicidade (kg.dia/mg); $H Q$ é o quociente de perigo (adimensional); RfD é a dose de referência (mg/kg.dia). Neste estudo, o 
nível tolerável de risco carcinogênico adotado foi de $10^{-5}$ (CETESB, 2006, 2007; CONAMA, 2009; ASTM, 2010). Para a análise de substâncias não carcinogênicas, quando a relação entre dose de ingresso e dose de referência for maior que 1 (um), existe perigo de efeitos adversos à saúde humana (USEPA, 1989a).

\section{Risco a partir da taxa de dose potencial variá- vel}

Como as concentrações dos contaminantes variam temporalmente no meio impactado, a quantidade dos compostos que entra em contato com o corpo humano não é constante ao longo do tempo. Dessa forma, a taxa de dose potencial para um tempo $t$ qualquer $\left(\mathrm{TDpot}_{\mathrm{t}} \mathrm{)}\right.$ ) pode ser calculada como o produto entre a concentração de exposição no tempo correspondente $\left(\mathrm{C}_{\mathrm{POE}, \mathrm{t}}\right)$ e a taxa de consumo de água subterrânea (IR*EF) (equação $4)$.

$$
\text { TDpot }_{t}=C_{P O E, t} \cdot I R \cdot E F \quad(\text { equação 4) }
$$

onde TDpot,$t$ é a taxa de dose potencial no tempo $t$ (mg/ano); $C_{P O E, t}$ é a concentração de exposição no tempo $t(\mathrm{mg} / \mathrm{L})$.

A dose potencial total (massa total ingerida, neste caso) do composto químico (Dpot ${ }_{\text {TOTAL }}$ ) para o tempo total de exposição (n) corresponde à somatória dos valores das taxas de doses potenciais recebidas durante vários intervalos de exposição $\left(E_{t}\right)$ (equação 5). A duração de exposição de cada concentração $\left(E D_{t}\right)$ é variável e, neste estudo, é equivalente ao intervalo de tempo entre as coletas de campo. Na modelagem matemática, o tempo total de exposição (n) deve representar a duração de exposição prevista para o receptor e uso de solo da metodologia adotada ( 25 anos para receptor industrial/comercial, por exemplo). No entanto, este estudo utilizou o tempo até a atenuação da pluma dos compostos dissolvidos, uma vez que após o tempo considerado, não há concentrações que incrementam no valor do risco total.

$$
\operatorname{Dpot}_{\text {TOTAL }}=\sum_{t=1}^{n} \text { TDpot }_{, t} \cdot E D_{t} \cdot \frac{10^{-3} g}{m g}
$$

onde Dpot $_{\text {TOTAL }}$ é a dose potencial total (g) para a duração de exposição; $n$ é a duração de exposição (anos); $E D_{t}$ é a duração de exposição para a con- centração no tempo $t$ (anos).

A partir das doses potenciais totais (equação 5) é possível calcular o risco total carcinogênico (equação 6) e não carcinogênico (equação 7) para o tempo de exposição considerado.

$$
\begin{aligned}
& R_{\text {TOTAL }}=\left[\frac{\text { Dpot }_{\text {TOTAL }}}{B W \cdot A T_{c}}\right] \cdot S F \quad(\text { equação 6) } \\
& H Q_{\text {TOTAL }}=\left[\frac{D p o t_{T O T A L}}{B W \cdot A T_{n c} \cdot R f D}\right] \quad(\text { equação 7) }
\end{aligned}
$$

onde $R_{\text {TOTAL }}$ e $H Q_{\text {TOTAL }}$ são os riscos carcinogênico e não carcinogênico totais, respectivamente (adimensional); $A T_{c}$ é o tempo médio para efeitos carcinogênicos (anos); $A T_{n c}$ é o tempo médio para efeitos não carcinogênicos (anos).

\section{RESULTADOS E DISCUSSÃO}

\section{Concentrações de exposição na área experi- mental}

Em ambientes subsuperficiais, processos de dissolução, transporte e biodegradação são responsáveis pela redução de massa e de concentrações dos poluentes ao longo do tempo (WIEDEMEIER et al., 1999). Concentrações de benzeno e tolueno medidas próximas da fonte (PM4) de um estudo de atenuação natural com gasolina brasileira demonstram a variação temporal das concentrações de exposição (Figura 2). Nos primeiros meses, as concentrações de benzeno e tolueno eram baixas devido à lenta dissolução destes compostos da fonte para a água subterrânea. Máximas concentrações na água subterrânea próximas à fonte (PM4) de benzeno e tolueno (respectivamente $11,41 \mathrm{mg} / \mathrm{L}$ e $21,71 \mathrm{mg} / \mathrm{L}$ ) foram atingidas aproximadamente 2,0 anos após a liberação controlada da gasolina. Durante esse tempo (até 2,0 anos), o etanol foi o substrato preferencial da biodegradação, inibindo a atenuação dos hidrocarbonetos monoaromáticos (CORSEUIL et al., 2011). Após 14,3 anos do início do experimento, as concentrações aquosas de benzeno e de tolueno na região da fonte já se encontravam abaixo de $1 \mu \mathrm{g} / \mathrm{L}$ (limite de detecção). Estes resultados demonstram que as máximas concentrações de exposição estiveram presentes na área durante um pequeno intervalo de tempo da duração de exposição considerada. 


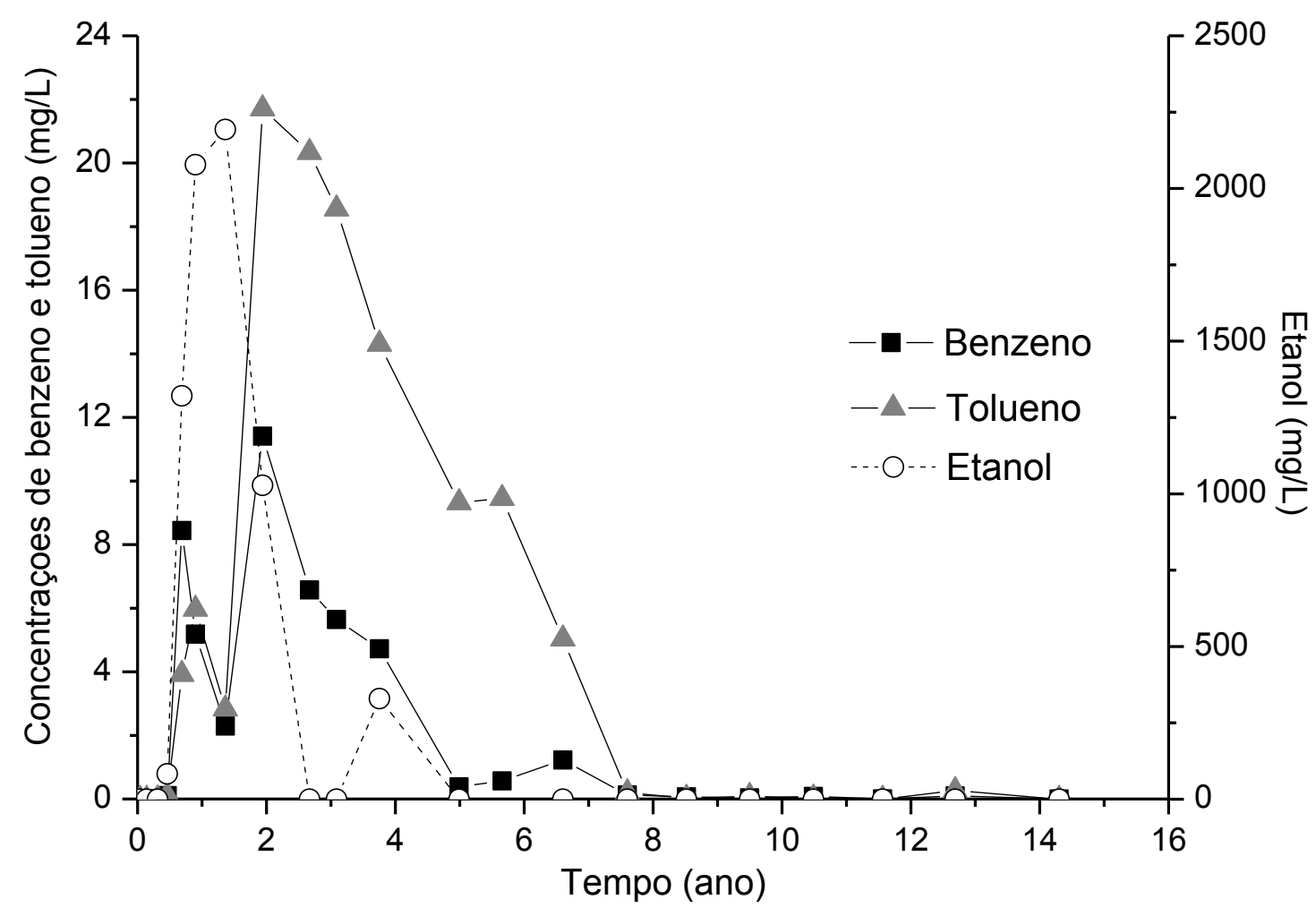

Figura 2 - Concentrações de exposição de benzeno ( $\longrightarrow-$ ), tolueno ( - - ) e de etanol ( - - O- - ) no poço de monitoramento PM4 da área experimental da Atenuação Natural Monitorada (ANM)

Figure 2 - Exposure concentrations of benzene ( -- ), toluene ( $-<-$ ) and ethanol ( - - - - ) in monitoring well PM4 experimental area of Monitored Natural Attenuation (ANM)

\section{Taxa de dose potencial variável}

A taxa de dose potencial $\left(\right.$ TDpot $\left._{, t}\right)$ é definida como a quantidade de produto químico ingerido por unidade de tempo (USEPA, 1992b). Sendo as concentrações dos contaminantes variáveis temporalmente, a ingestão destes compostos, associada ao cenário de contaminação, é um processo variável em vez de contínuo (USEPA, 1992b). As taxas de dose potencial (equação 4) de benzeno e tolueno foram estimadas a partir do produto entre as concentrações de exposição nos diversos tempos de monitoramento (Figura 2) e a taxa de consumo de água subterrânea (IR*EF) (Tabela 1). As maiores taxas de dose potencial (poço PM4) de benzeno (2.852,8 mg/ano) e de tolueno (5.426,5 mg/ano) foram atingidas no tempo amostral de 1,9 anos (Figura 3), tempo em que as máximas concentrações estiveram presentes. Nos primeiros meses do experimento, e após 8 anos da liberação do combustível, as taxas de dose potencial dos compostos químicos de interesse foram insignificantes devido às baixas concentrações presentes desses contaminantes na água subterrânea. Estes resultados demonstram como a taxa de dose potencial variou expressivamente ao longo dos anos, mesmo na região próxima à presença de fase livre.
A dose potencial total ingerida ( Dpot $_{\mathrm{TOTAL}}$ ) (i.e., massa total ingerida) do composto químico ao longo do tempo de exposição pode ser representada como a soma das taxas de doses potenciais recebidas durante vários eventos de exposição (USEPA, 1992b; WHO, 1999). A partir dos valores de taxa de dose potencial variável do benzeno e do tolueno, e de suas respectivas durações de exposição $\left(E_{t}\right)$ (Figura 3), foi possível determinar a dose potencial total de cada contaminante na área de estudo (equação 5). Desde o início do experimento até a completa exaustão dos contaminantes, a dose potencial total ingerida de benzeno foi de $5,9 \mathrm{~g}$, e de tolueno, de 17,9 g (para um receptor próximo à fonte - PM4) (Figura 3).

Aplicando-se a metodologia RBCA, o cálculo da dose potencial total seria realizado a partir de um único valor de taxa de dose potencial que se mantém constante, e dependente do tempo amostral. Por exemplo, as doses potenciais totais, considerando as concentrações dos contaminantes nos tempos amostrais de 0,5 anos, 1,9 anos e 5,0 anos e os parâmetros de exposição IR, EF, ED (Tabela 1) indicariam uma quantidade total ingerida de benzeno, respectivamente de $0,7 \mathrm{~g}, 71,3 \mathrm{~g}$ e $2,4 \mathrm{~g}$. Para o tolueno, as doses potenciais totais 
seriam de $0,74 \mathrm{~g}(\mathrm{em} \mathrm{t}=0,5$ anos), $135,7 \mathrm{~g}(\mathrm{t}=$ 1,9 anos) e 58,3 $\mathrm{g}(\mathrm{t}=5,0$ anos). Desta forma, a dose potencial total a partir da metodologia convencional de risco à saúde humana pode ser muito superior ou inferior à dose real ingerida dos compostos químicos, dependendo do tempo amostral analisado.

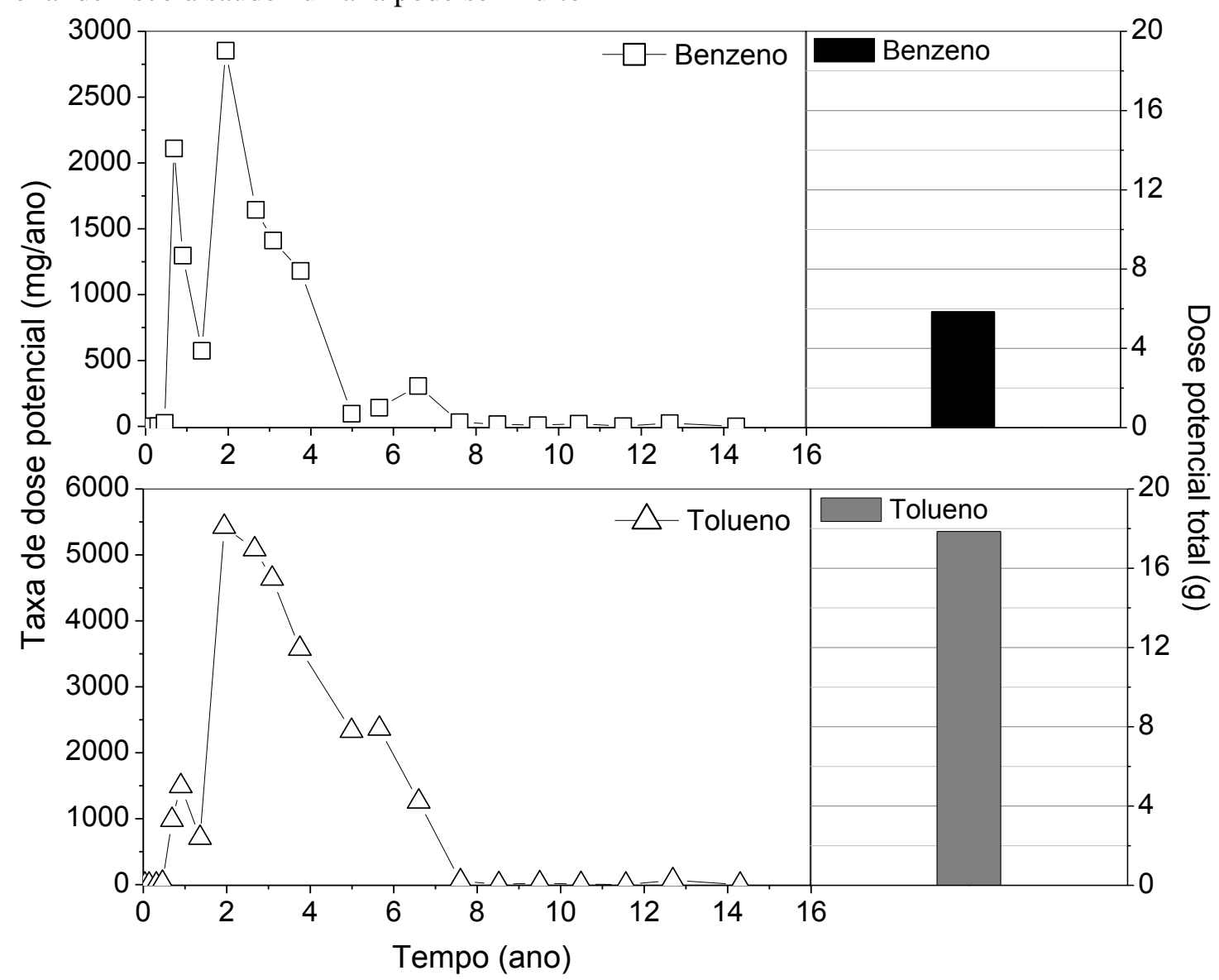

Figura 3 - Taxas de dose potencial variável e doses potenciais totais de benzeno e de tolueno na região da fonte (poço PM4) da área experimental da Atenuação Natural Monitorada (ANM)

Figure 3 - Variable potential dose rates and total potential doses of benzene and toluene in the source region (well PM4) of the experimental area of Monitored Natural Attenuation (ANM)

\section{Risco total}

O risco à saúde humana está condicionado à quantidade total do contaminante ingerida ao longo do tempo de exposição. As doses potenciais totais de benzeno e tolueno permitiram quantificar os riscos totais para essas substâncias no poço de monitoramento PM4 (equações 6 e 7). O risco carcinogênico total do benzeno foi de $1,8 \times 10^{-4}$, enquanto que para o tolueno, o risco não carcinogênico total foi de $3,5 \times 10^{-1}$. Considerando a metodologia RBCA e as doses potenciais totais apresentadas no item anterior, o risco carcinogênico para o benzeno seria de $2,0 \times 10^{-5}(\mathrm{t}=0,5$ anos $)$, $2,2 \times 10^{-3}(\mathrm{t}=1,9$ anos $)$ e $7,3 \times 10^{-5}(\mathrm{t}=5,0$ anos $)$, e o risco não carcinogênico para o tolueno seria de $1,4 \times 10^{-2}(\mathrm{t}=0,5$ anos $), 2,7 \times 10^{\circ}(\mathrm{t}=1,9$ anos $)$ e $1,1 \times 10^{0}(\mathrm{t}=5,0$ anos). Os maiores valores de risco (carcinogênico e não carcinogênico) obtidos para o estudo de caso pela metodologia RBCA ocorrem no intervalo de tempo onde as maiores doses potenciais totais dos contaminantes estari- am presentes $(t=1,9$ anos). Como a quantificação da dose potencial total pela metodologia convencional está condicionada a uma única e constante taxa de dose potencial, que por sua vez é dependente do tempo monitorado, estes valores conservadores de risco à saúde humana somente seriam obtidos caso esta avaliação fosse realizada aproximadamente 2 anos após a liberação do produto na água subterrânea, ou seja, exatamente no momento onde as maiores concentração de poluentes estiveram presentes na área investigada.

\section{CONCLUSÕES}

Este trabalho discutiu o conceito de temporalidade na quantificação do risco à saúde humana, utilizando um experimento de campo com liberação controlada de gasolina brasileira, monitorado por mais de 14 anos. Observou-se que a abordagem convencional de cálculo de risco, que 
considera a exposição aos receptores através de uma concentração constante ao longo de toda a duração de exposição, não refletiu as variações das concentrações dos contaminantes ocorridas desde o início da sua liberação na água subterrânea até a sua completa exaustão. Os resultados do experimento de campo da atenuação natural monitorada (ANM) mostraram que as máximas concentrações de exposição de benzeno e tolueno estiveram presentes durante um curto intervalo de tempo se considerada uma duração de exposição de 25 anos. Por essa razão, os valores de risco calculados pela metodologia RBCA apresentaram diferenças de duas ordens de grandeza, dependendo do tempo amostrado. Como a quantificação do risco, na maioria dos casos, não é baseada em dados históricos da área, mas sim em amostragens únicas e aleatórias, a concentração máxima da área em um dado tempo amostral não necessariamente fornecerá valores de risco conservadores. Embora seja um objetivo das metodologias de avaliação de risco assegurar a proteção à saúde do indivíduo e do meio ambiente, cuidados devem ser tomados pelos órgãos ambientais e responsáveis em relação às incertezas no cálculo do risco à saúde humana baseado em um único monitoramento.

Os dados apresentados neste trabalho confirmaram, com um estudo de campo, a influência da variação das concentrações na quantificação do risco. A utilização da taxa de dose potencial variável como alternativa complementar para o cálculo de risco à saúde humana, especialmente no caso da gasolina brasileira, vai depender da sua aplicação em modelos matemáticos que simulem os processos de transporte e transformação dos compostos aromáticos, e levem em consideração a presença de biocombustíveis. Desta forma, a priorização das ações de intervenção/remediação em uma área impactada poderá considerar a avaliação temporal da taxa de dose potencial.

A Universidade Federal de Santa Catarina desenvolveu em parceria com a Petrobras um software (Solução Corretiva Baseada no Risco SCBR) que permite aplicar esses conceitos para as peculiaridades da gasolina brasileira. O SCBR tem sido utilizado experimentalmente como ferramenta de gestão em postos de combustíveis, terminais, refinarias e áreas de exploração e produção onshore da Petrobras. Nestas condições, a disponibilidade de um procedimento complementar de cálculo de risco à saúde humana com foco na variação da taxa de dose potencial, para a duração de exposição prevista na metodologia adotada, será possível aplicá-lo tanto em locais onde não existem contaminações (caráter preventivo), como em cenários em que a contaminação está presente e se deseja comparar a exposição presente e seus efeitos no futuro. Deste modo, valores de risco de acordo com as condições de exposição específicas do local e particularidades dos produtos poderão ser obtidos, reduzindo as incertezas na quantificação do risco no que se refere à escolha da concentração representativa da área e auxiliando no processo de tomada de decisão na definição de tecnologias mais adequadas para recuperação de áreas impactadas.

Recomenda-se que sejam ampliados estudos na determinação de valores orientadores que considere a taxa de dose potencial variável, visto que os valores referência são derivados a partir de metodologias de avaliação de risco à saúde humana, e cenários genéricos de exposição.

\section{AGRADECIMENTOS}

Ao Conselho Nacional de Desenvolvimento Científico e Tecnológico (CNPq) pela concessão da bolsa de mestrado do primeiro autor, e ao Centro de Pesquisas e Desenvolvimento Leopoldo Américo Miguez de Mello - CENPES (Petrobras) pelo financiamento dos estudos de campo.

\section{REFERÊNCIAS}

AFCEE. Handbook for Remediation of Petroleum - Contaminated Sites (A Risk-Based Strategy). Air Force Center for Environmental Excellence Technology, Brook AFB: Texas, 1998.

ANDRICEVIC, R.; SRZIC, V.; GOTOVAC, H. Risk characterization for toxic chemicals transported in aquifers. Advances in Water Resources, v. 36, p. 86-97, 2012.

ASTM. Standard Guide for Risk-Based Corrective Action: American Society for Testing and Materials - E 2081-
00. 2000

ASTM. Standard Guide for Risk-Based Corrective Action Applied at Petroleum Release Sites: American Society for Testing and Materials - E 1739-95-Reapproved 2010. 2010.

BACIOCCHI, R., BERARDI, S.; VERGINELLI, I. Human health risk assessment: Models for predicting the effective exposure duration of on-site receptors exposed to contaminated groundwater. Journal of Hazardous. Materials, v. 181, p. 226-233, 2010. 
BIÉN, J.D.; ter MEER, J.; RULKENS, W.H.; RIJNAARTS, H.H.M. A GIS-based approach for the long-term prediction of human health risks at contaminated site. Environmental Modeling and Assessment, v. 9, p. 221-226, 2004.

CETESB. Manual de Gerenciamento de Áreas Contaminadas. Companhia Ambiental do Estado de São Paulo. $2^{\mathrm{a}}$ ed. São Paulo: CETESB, 2001. 612p.

CETESB. Anexo VII - Decisão de Diretoria no 010/2006/C. Ações corretivas baseadas em risco (ACBR) aplicadas a áreas contaminadas com hidrocarbonetos derivados de petróleo e outros combustíveis líquidos - procedimentos. Companhia Ambiental do Estado de São Paulo, São Paulo, 2006.

CETESB. Companhia Ambiental do Estado de São Paulo. Decisão de Diretoria $n^{0} 103 / 2007 / C / E$. Dispõe sobre o procedimento para o gerenciamento de áreas contaminadas. São Paulo, 2007. 40p.

CHEN, B. Water pollution simulation and health risk assessment through a refined contaminant transport model. Water Air Soil \& Pollution, v. 200, p. 323-339, 2009.

CNEN. Diretrizes Básicas de Proteção Radiológica. Comissão Nacional de Energia Nuclear - CNEN-NN-3.0. Rio de Janeiro: CNEN, 2005. 27p.

COLONESE, B. L. Aplicação do modelo SCBR no gerenciamento de áreas contaminadas - estudo de caso: terminal de petróleo de São Sebastião. Florianópolis, Santa Catarina, 2010. 143p. Dissertação (Mestrado em Engenharia Ambiental). Universidade Federal de santa Catarina.

CONAMA. Resolução no ${ }^{\circ}$ 420, de 28 de dezembro de 2009. Dispõe sobre critérios e valores orientadores de qualidade do solo quanto à presença de substâncias químicas e estabelece diretrizes para o gerenciamento ambiental de áreas contaminadas por essas substâncias em decorrência de atividades antrópicas. Conselho Nacional do Meio Ambiente. Ministério do Meio Ambiente. 2009. 16p.

CONNOR, J.A.; BOWERS, R. L.; MCHUGH, T. E.; SPEXET, A. H. RBCA Tool Kit for Chemical Releases Software Guidance Manual. Version 2. Houston, Texas: GSI Environmental Inc, 2007. 120p.

CORSEUIL, H. X.; SCHNEIDER, M. R.; ROSÁRIO, M.; MALISKA JR, M. Solução Corretiva Baseada no Risco (SCBR): Modelo matemático de tomada de decisão para o gerenciamento ambiental de águas subterrâneas contaminadas. In: CONGRESSO BRASILEIRO DE ÁGUAS SUBTERRÂNEAS, 14, Curitiba, 2006. Anais... São Paulo, Associação Brasileira de Águas Subterrâneas, 2006. n.p.

CORSEUIL, H. X.; MONIER, A. L.; FERNANDES, M.; SCHNEIDER, M. R.; NUNES, C.C.; ROSÁRIO, M.; ALVAREZ, P.J.J. BTEX Plume Dynamics Following an Ethanol Blend Release: Geochemical Footprint and Thermodynamic Constraints on Natural Attenuation. Environmental Science \& Technology, v. 45, p. 3422-3429, 2011.

IAEA. Near surface disposal of radioactive waste - Safety Standards Series. International Atomic Energy Agency No. WS-R. Vienna: IAEA, 1999. 42p.

IAEA. Safety assessment methodologies for near surface disposal facilities - Results of a co-ordinated research project. Volume 1 - Review and enhancement of safety assessment approaches and tools. International Atomic Energy Agency. Vienna: IAEA, 2004a. 413p.

IAEA . Safety assessment methodologies for near surface disposal facilities - Results of a co-ordinated research project. Volume 2 - Test case. International Atomic Energy Agency. Vienna: IAEA, 2004b. 338p.

IRIS. A - Z List of Substances. Integrated Risk Information System, 2009. Disponível em: <http://cfpub.epa.gov/ncea/iris/index.cfm?fuseaction=iris.sho wSubstanceList>. Acesso em 03 jul. 2012.

NEWELL, C. J.; McLEOD, R.K.; GONZALES, J.R. BIOSCREEN - Natural Attenuation Decision Support System. Cincinnati, Ohio: United States Environmental Protection Agency, 1996. 100p.

NRC. Risk assessment in the Federal Government: managing the process. National Research Council. Washington: National Academy Press, 1983. 191p.

PONTEDEIRO, E. M. B. D.; HEILBRON FILHO, P. F. L.; COTTA, R.M. Assessment of the Mineral Industry NORM/TENORM Disposal in Hazardous Landfills. Journal of Hazardous Materials, v. 136, p. 563-568, 2007.

PONTEDEIRO, E. M.; van GENUCHTEN, M.Th.; COTTA, R.M.; SIMUNEK, J. The effects of preferential flow and soil texture on risk assessments of a NORM waste disposal site. Journal of Hazardous Materials, v. 174, p. 648-655, 2010.

SCHNEIDER, A. C.; NUNES, C. C.; JOUSSEF, K. L.; SCHVEITZER JUNIOR, A.; SCHNEIDER, M. R.; ROSÁRIO, M.; CORSEUIL, H. X. SCBR model as a decision support system tool for management of biofuels contaminated sites. In: ENCONTRO LATINO AMERICANO DE CFD APLICADO À INDÚSTRIA DE ÓLEO E GÁS, 5, Rio de Janeiro, 2012. Rio de Janeiro, Engineering Simulation and Scientific Software-ESSS, 2012.

SPENCE, L. R.; WALDEN, T. RISC4 - Risk-Integrated Software for Clean-Ups.User's Manual. Pleasanton, CA: BP Oil International, 2001. 463p.

USEPA. Superfund Exposure Assessment Manual. United States Environmental Protection Agency - EPA/540/188/0012. Washington, D.C: United States Environmental Protection Agency, 1988.

USEPA. Risk Assessment Guidance for Superfund - Vol. 1: Human Health Evaluation Manual - Part A. United States Environmental Protection Agency -EPA/540/1-89/002 .Washington, D.C: United States Environmental Protection Agency, 1989a.

USEPA. Exposure Factors Handbook. United States Environmental Protection Agency - EPA/600/8-89/043. Washington, D.C: United States Environmental Protection Agency, 1989b.

USEPA. Supplemental Risk Assessment Guidance for the Superfund Program. United States Environmental Protection Agency - EPA/901/5-89/001. Washington, D.C: United States Environmental Protection Agency, 1989c.

USEPA. Supplemental Guidance to RAGS: Calculating the Concentration Term. United States Environmental 
Protection Agency - PB92 - 963373. Washington, D.C: United States Environmental Protection Agency, 1992a.

USEPA. Guidelines for Exposure Assessment. United States Environmental Protection Agency - EPA/600/Z92/001. Washington, D.C: United States Environmental Protection Agency, 1992b.

USEPA. Determination of Inorganic Anions by Ion Chromatography. United States Environmental Protection Agency - Method 300.0. Cincinnati, Ohio: United States Environmental Protection Agency, 1993.

USEPA. Carcinogenic Effects of Benzene: An Update. United States Environmental Protection Agency EPA/600/P-97/001F. Washington, D.C: United States Environmental Protection Agency, 1998.

USEPA. Calculating Upper Confidence Limits for Exposure Point Concentrations at Hazardous Waste Sites.
United States Environmental Protection Agency - OSWER 9285.6-10. Washington, D.C: United States Environmental Protection Agency, 2002.

USEPA. Toxicological Review of Toluene. United States Environmental Protection Agency - EPA/635/R-05/004. Washington, D.C: United States Environmental Protection Agency, 2005.

WIEDEMEIER, T. H.; RIFAI, H. S.; NEWELL, C. J.; WILSON, J. T. Natural Attenuation of Fuels and Chlorinated Solvents in the Subsurface. 1.ed. New York, John Wiley \& Sons, Inc., 1999. 244p.

WHO. Principles for the Assessment of Risks to Human Health from Exposure to Chemicals. Environmental Health Criteria 210. Switzerland, Geneva: World Health Organization, 1999. 